\title{
Aplicación Integrada para Construcción de PCBs mediante Tecnología CNC y Ajuste Automático a la Superficie
}

\author{
Jared Roberto Ocampo \\ Iván de Jesús Deras ${ }^{1}$ \\ Universidad Tecnológica Centroamericana (UNITEC), San Pedro Sula, Honduras
}

(Enviado: Mayo, 2014)

\begin{abstract}
Resumen:
Uno de los mayores problemas que tienen los alumnos de las carreras del área electromecánica de Unitec es producir sus propios tableros de circuitos impresos (PCB) de manera precisa y segura. Actualmente los estudiantes utilizan la técnica de ataque químico y transferencia de tóner para realizar sus prototipos de PCBs en clases de circuitos eléctricos y electrónica. Sin embargo, esta técnica a pesar de ser de fácil aplicación, es larga y tediosa, produce placas con defectos cuando las pistas son delgadas y es peligrosa por causa del ácido que debe usarse, el cual es nocivo para la salud y el medioambiente. A través de este proyecto se creó un sistema informático integrado que permite hacer uso de una maquina CNC para producir tableros de circuito tomando en consideración la altura de las placas de manera más rápida, con menos defectos de fabricación y sin la necesidad de poner en peligro la salud de los usuarios y de contaminar el medioambiente por el uso de ácidos nocivos.
\end{abstract}

Palabras Claves: PCB, Circuito Impreso, Prototipado Electrónico, CNC.

\begin{abstract}
:
One of the biggest problems facing students of the electromechanical majors at Unitec is to produce their own printed circuit boards (PCBs) accurately and safely. Currently students use the etching and ink transfer technique to build the prototypes they use in their circuits and electronic classes. However, this technique despite being easy to apply, is long and tedious, produces boards with defects (when the tracks are thin) and is dangerous due to the utilized acid which is harmful to health and the environment. Through this project, an integrated computer system was created which allows the use of a CNC machine to produce circuit boards. Such an approach allows the height of the board to be considered more quickly, while incurring fewer manufacturing defects and avoiding the endagerment of the health of users and the pollution of the environment with harmful acids
\end{abstract}

Keywords: PCB, Printed Circuit Board, Electronic Prototyping, CNC.

* Los autores agradecen a Yasbin Sabillón, Manuel Fernández, José Guzmán, Carlos Morales, Miklos Szabo y Jhonan Pabón quienes apoyaron con información, sugerencias y pruebas prácticas sin las cuales hubiera sido muy difícil llevar a cabo este trabajo de investigación y su correspondiente artículo.

\section{Introducción}

En la actualidad aun las empresas más pequeñas cuentan con maquinaria automática basada en electrónica que permite que las operaciones y procesos de producción sean más eficientes y que las empresas se vuelvan mucho más competitivas. Para dar respuesta a la creciente necesidad de profesionales que no solo manejen y reparen estos equipos de hardware y software, sino que tambien puedan diseñar equipo hecho a la medida, fue necesario que las universidades dieran un paso adelante con carreras que satisfacieran esta necesidad. Carreras tales como Ingeniería en Sistemas Electrónicos, Mecatrónica y

\footnotetext{
${ }^{1}$ Autor para correspondencia. Email: ideras@unitec.edu
} 
Biomédica (denominadas "Ingenierías Electromecánicas" y ofrecidas en UNITEC), son algunas de las que dan respuesta a esta necesidad.

Debido a que la electrónica es el fundamento de la mayoría de los sistemas de control automático, uno de los tópicos obligados para todas estas carreras es el diseño de sistemas electrónicos. El tema puede verse de forma teórica y hacerse demostraciones mediante el uso de simuladores virtuales tales como NI Multisim, CircuitMaker y CadSoft Eagle. Sin embargo, en los últimos años se ha aprendido que el formato de enseñanza magistral en donde el maestro habla y el alumno escucha provee pocas oportunidades de involucrar de forma activa a los estudiantes en la resolución de problemas del mundo real y de esta forma aumentar su interés y motivación. Esta técnica tradicional de enseñanza ha sido conectada a baja motivación estudiantil y a su vez, la motivación ha sido conectada al aprendizaje del estudiante y a la asistencia a clases (Bonwell \& James, 1991 y Devadoss $\&$ Foltz, 1996). Por lo tanto, para que este tema pueda ser entendido adecuadamente por los alumnos y se vuelva un medio de motivación en lugar de uno de disuasión, es necesario ir más allá del conocimiento teórico y de los simuladores computarizados, y permitirles construir prototipos que provean al estudiante con experiencias prácticas valiosas.

Una forma de introducir esta experiencia "hands-on" (práctica) en las clases antes mencionadas es promoviendo que los alumnos puedan crear sus propios tableros de circuitos impresos (PCB por sus siglas en inglés), y que ensamblen y suelden los componentes electrónicos correspondientes para que prueben en el mundo real sus diseños. Esto les permitirá concebir una idea para resolver un problema específico, diseñar una solución que dé respuesta al desafío, implementen en software y hardware mediante prototipos el diseño realizado y finalmente lo operen bajo condiciones reales para que validen que tan bien su diseño se comporta y da respuesta al problema propuesto. De acuerdo a Wee (2013), el hacer uso de este modelo educativo debería convertir el aprendizaje de ingeniería en una actividad más emocionante y relevante, proveyendo a los estudiantes con oportunidades para desarrollar habilidades interpersonales, así como usar su conocimiento técnico y razonamiento para resolver problemas del mundo real.
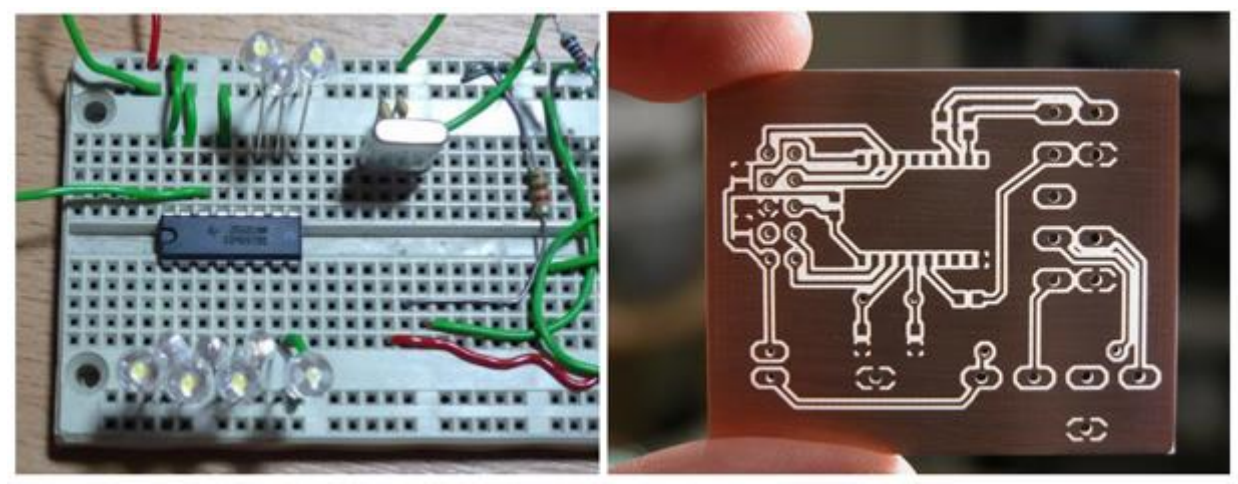

Figura 1. Placa de pruebas (Protoboard) y tablero de circuito impreso (PCB)

Una forma de construir prototipos rápidos de circuitos es mediante el uso de placas de pruebas (en inglés protoboard o breadboard). Como se observa en la Figura 1, este dispositivo es un tablero con orificios que están eléctricamente conectados unos con otros y en donde se pueden insertar componentes electrónicos y cables para conectar aquellos componentes que no se interconectan internamente a través de la placa. Una de sus 
ventajas más grandes es la facilidad con la que se pueden colocar y quitar los componentes del circuito que se desea probar. Sin embargo, este tipo de placas solo sirven para desarrollar circuitos temporales que luego necesitan ser montados en un tablero de circuitos impreso (PCB) para su uso permanente (ver Figura 1).

Una de las habilidades que los alumnos del área de electromecánicas deben desarrollar es como pasar de una placa de pruebas temporal a un tablero de circuito permanente. La técnica usada tradicionalmente para fabricar PCB de manera manual es el atacado químico combinado con la transferencia de tóner, debido a que no requiere de materiales difíciles de encontrar y es por lo tanto muy accesible. Sin embargo entre los problemas que presenta esta técnica son los diversos pasos y diferentes elementos requeridos que hacen que el tiempo para crear una PCB sea elevado. Además, esta técnica nos es capaz para producir pistas delgadas $(<2 \mathrm{~mm})$ debido a los problemas relacionados con la transferencia de tóner en sectores muy estrechos. Esta situación hace que sea imposible para los alumnos trabajar con componentes electrónicos de la tecnología de montaje superficial (en inglés surface-mount technology o SMT) debido a que estos requieren de pistas sumamente delgadas. Finalmente, esta metodología requiere del uso de ácidos para atacar químicamente y debido a que estos químicos son nocivos para los seres humanos y el medioambiente, esta técnica de fabricación no es ecológica ni sostenible.

La metodología de producción de PCBs usando CNC (Machined Circuit Boards o MCB) puede reducir el tiempo de fabricación, mejorar la precisión de los tableros de circuitos y eliminar la necesidad de usar ácidos nocivos para la salud y el medioambiente. Por lo tanto, el objetivo principal de este proyecto fue crear una aplicación de software que integre todos los pasos y aplicaciones informáticas necesarias para la fabricación de PCBs mediante tecnología CNC en una sola herramienta informática de fácil uso.

\section{Marco Teórico}

De acuerdo a Groover (1997), un tablero de circuitos impresos (PCB), es "un panel plano chapeado con material aislante, diseñado para proporcionar conexiones eléctricas entre los componentes electrónicos que se encuentran en él." Los tableros que son usados están formados por un sustrato aislante con revestimiento de cobre que es usado para realizar las conexiones eléctricas entre los componentes mediante pistas delgadas de este material conductor. El espesor del sustrato está en un rango entre 0.8 y $3.2 \mathrm{~mm}$ y el de la capa de cobre aproximadamente $0.04 \mathrm{~mm}$. Existen tres tipos especiales de tableros de circuitos impresos, los de un solo lado (la capa de cobre está en un solo lado del sustrato), los de dos lados y los de multicapas (capas alternadas de elemento conductor y aislante). Por supuesto, los más usados son los de uno y dos lados.

La producción de tableros de circuitos impresos que estén listos para el ensamble de componentes requiere de varias operaciones de procesamiento. Entre ellas se encuentran la limpieza, el perforado de los orificios de conexión, el copiado de los patrones de las pistas y el ataque químico. Si bien es cierto la producción industrial de estos tableros requiere de ciertas condiciones específicas, dado que el alcance de este proyecto está orientado a la producción de placas por parte de estudiantes de colegio y universitarios, a continuación se describe como producir PCBs para uso cotidiano y de forma no industrial, las cuales serán denominadas PCB estudiantiles. 


\subsection{Fabricación de tableros mediante ataque químico}

De acuerdo a las instrucciones de Groover (1997) y con los ajustes propuestos por Fernando Rey (2013), la fabricación de PCBs estudiantiles mediate ataque químico y transferencia de tóner requiere los siguientes pasos:

\section{Preparación del tablero}

La preparación inicial del panel que se convertirá en un PCB incluye el cortado del tablero a un tamaño adecuado (usualmente usando una sierra de marco), las perforaciones y el proceso de limpieza. Las perforaciones son de dos tipos, los orificios para alinear los tableros correctamente y las perforaciones funcionales que se utilizan para insertar terminales, para conectar ambos lados del tablero en el caso de tableros con dos caras y sujetar ciertos componentes. El proceso de limpieza es necesario ya que pequeñas partículas de suciedad o grasa en la superficie del tablero pueden causar defectos en el patrón de circuitos. Estos problemas se deben a que la suciedad y grasa pueden evitar la transferencia del patrón al tablero o inhibir el ataque químico. Por lo tanto, después que se quitan todas las rebabas producidas por el corte de la placa (usando una lija gruesa), la superficie de la placa se pule ligeramente con una lija fina húmeda para que el tóner pueda adherirse mejor al cobre. Para eliminar bien todas las impurezas se sugiere mojar la placa con agua y limpiarla con un paño limpio o papel toalla. Luego se aconseja repetir el proceso de limpieza pero esta vez usando alcohol isopropilíco hasta que el papel o trapo queda limpio luego de pasarlo por la superficie de la placa. Una vez la placa está limpia es importante no tocarla con los dedos para evitar trasladar grasa e impurezas al cobre.

Copia del patrón de circuitos

Con el propósito de crear una máscara que sirva de recubrimiento y protección ante el agente químico que atacará el cobre, es necesario copiar el patrón de circuitos que se desea crear en la superficie del cobre. Al hacer esto se asegura que el químico removerá todo el cobre que no esté protegido creando así las pistas e islas del circuito. En este caso, el patrón es copiado mediante la impresión del diseño en papel fotográfico usando una impresora láser. El tóner actúa como material resistente al acido, protegiendo las áreas que no deben de ser corroídas.

Para fijar el tóner a la placa de cobre se usa una plancha bien caliente sin vapor. La hoja de papel fotográfico con la impresión del circuito se coloca hacia abajo (con la tinta haciendo contacto con la placa de cobre) y se plancha de forma cuidadosa de manera que por acción del calor el tóner se empieza a desprender y se pega al cobre. Una vez finalizado el planchado, la placa se coloca en un recipiente con agua para que el papel se desprenda. Si quedaran residuos de papel pegados, estos pueden retirarse usando de manera cuidadosa un cepillo de dientes mojado o un punzón fino. Las áreas dañadas o donde el tóner no se adhirió correctamente pueden ser repasadas usando un marcador punta fina indeleble para asegurar que todas las pistas e islas quedan cubiertos.

\section{$\underline{\text { Ataque químico }}$}

El ataque químico es el paso de la secuencia que transforma la película de cobre sólido en interconexiones de un circuito eléctrico. Este proceso puede usar diferentes químicos, aunque el ácido clorhídrico o ácido muriático y el agua oxigenada son de los más usados. 
El ácido clorhídrico es corrosivo y tóxico por inhalación y contacto y deben de tomarse precauciones para evitar daños a la salud de quien lo usa. El ácido clorhídrico y el agua oxigenada se mezclan usando cantidades iguales, y se colocan en un recipiente plástico lo suficientemente grande como para cubrir la placa a grabar. Al introducir la placa de cobre en la mezcla realizada se observara que el conjunto toma un color verde por causa de la reacción química. La operación de atacado químico se puede acelerar moviendo gentilmente el recipiente para hacer olas, teniendo cuidado de no salpicar y tener algún accidente. Todo el proceso dura unos cinco minutos en total. Al final la placa se retira de la mezcla de ácido clorhídrico y agua oxigenada y se coloca en un recipiente con agua limpia. El proceso de limpieza final requiere del uso de una lija y de agua limpia para poder ir descubriendo de a poco las pistas creadas por el proceso.

Una vez que la placa está lista se procede a taladrar los agujeros en donde se montarán los componentes electrónicos, esto se hace usando como guia las islas creadas en la placa. Usualmente se usa un Dremel con brocas de $0.7 \mathrm{~mm}$ (componentes), $1 \mathrm{~mm}$ (conectores y puentes) y $1.5 \mathrm{~mm}$ (bornes). Una vez insertadas los componentes, sus terminales se sueldan en los orificios usando estaño.

\subsection{Fabricación de tableros usando CNC}

Debido a que los tableros de circuitos están compuestos de un sustrato no conductor revestido por una superficie de cobre, otra forma de crear las pistas necesarias para el circuito es removiendo el material excedente mediante una operación de fresado. Para lograr la precisión necesaria esto puede hacerse utilizando una máquina de control numérico computarizado (CNC). El control numérico se usa para automatizar máquinas y herramientas de manera que pueden controlarse a través de comandos programados usando un lenguaje denominado Código G (en inglés G-Code). Este código brinda instrucciones al controlador de movimientos sobre la forma en que cada eje de la fresadora debe de moverse (Lynch, 2013).

Pasos y software usados para fabricar MCBs

A pesar que el fresado mediante tecnología CNC incrementa la velocidad de manufactura y mejora la precisión y repetitividad de las piezas creadas, en el caso de la creación de tableros de circuitos son varios los pasos que son requeridos para su fabricación. Siguiendo la metodología propuesta por Fritz (2010), como puede verse en la Figura 2, la fabricación de tableros de circuitos usando CNC puede realizarse siguiendo 4 pasos:

1. Se produce el diseño del layout del tablero de circuito que se quiere producir en formato Gerber, que es un código numérico usado para la fabricación de tarjetas electrónicas (Williams, 2004). Para esto es necesario hacer uso de un programa tal como Cadsoft Eagle, PCB Artist, KiCad, etc.

2. El archivo Gerber es entonces introducido a un programa de manufactura asistida por computadora (en inglés computer aided manufactring o CAM) que calcula las rutas que debe seguir la herramienta usada por la máquina fresadora (en inglés toolpaths) a partir del layout del circuito descrito en el archivo Gerber. Para esto puede usarse el programa "pcb2gcode" escrito originalmente por Jeff Protheros en lenguaje $\mathrm{C}$ a partir del programa "gerber2gcode" y reescrito más adelante en $\mathrm{C}++$ por Patrick Birnzain (SourceForge, 2011).

3. El software CAM genera entonces un archivo con instrucciones para la máquina fresadora $\mathrm{CNC}$ en código-G, y este es introducido para la fabricación del tablero 
de circuito mecanizado (MCB). Algunos ejemplos de controladores populares que pueden controlar maquinas $\mathrm{CNC}$ a partir del código $\mathrm{G}$ son el EMC2 (ahora llamado LinuxCNC) y el Mach 3.

4. Se monta y sujeta el tablero en la máquina fresadora y se ejecuta el programa en código-G producido para poder fabricar el circuito.

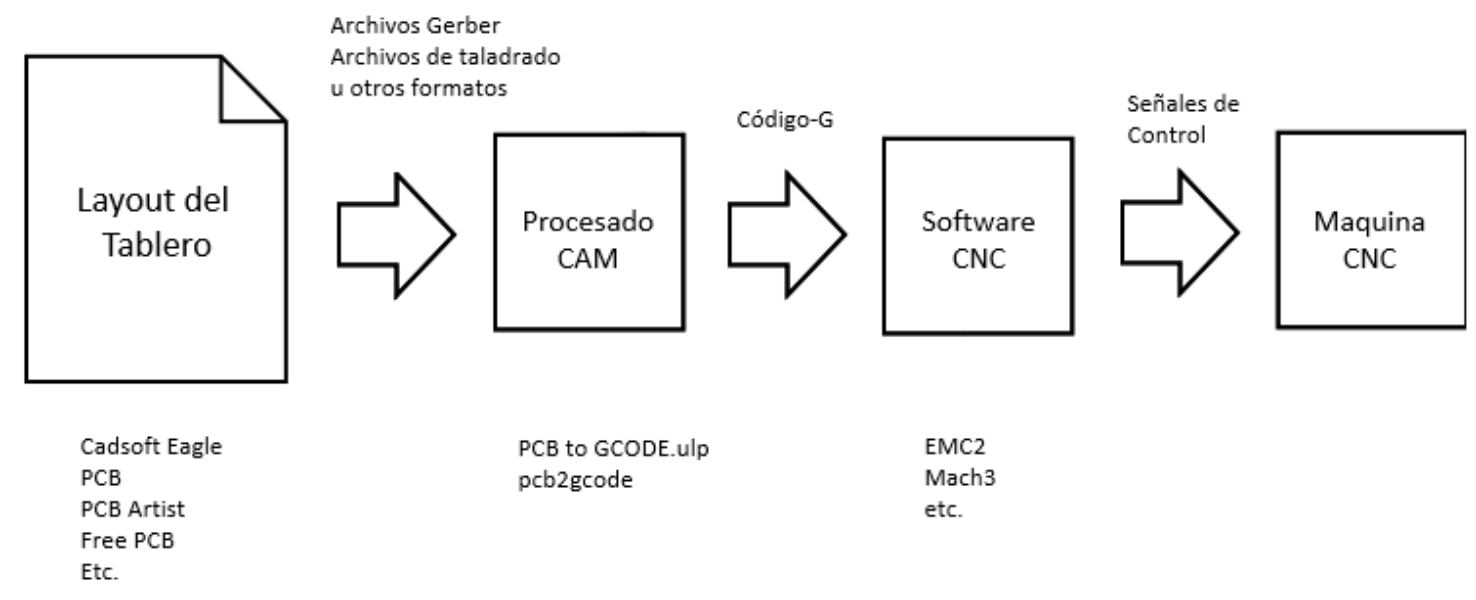

Figura 2. Pasos de la metodología seguida para fabricar una MCB

Para poder producir MCBs entonces se requiere de una máquina fresadora CNC cartesiana, robusta y preferiblemente con una velocidad de husillo alta $(10,000 \mathrm{rpm}$ o mejor), una computadora con controlador para la máquina $\mathrm{CNC}$, herramientas de corte (fresas) con diámetros suficientemente pequeños para lograr la precisión requerida, elementos de sujeción segura, diseño del tablero de circuito a producir (layout de la placa electrónica) en formato Gerber y software para transformar este diseño a código G para su posterior mecanizado usando la máquina $\mathrm{CNC}$.

Corrección de problemas de alineación y sujeción del tablero

Uno de los problemas potenciales que tienen los MCBs es que requieren de un control preciso de la altura de la placa. Esto se debe a que el mecanizado $\mathrm{CNC}$ requiere especificar la profundidad de corte, y si la altura de la placa no es constante podría haber lugares en donde no se remueva el material por causa de una profundidad de corte insuficiente (dejando pistas e islas sin terminar), o en donde se genere un corte demasiado profundo (desgastando innecesariamente las herramientas de corte). Este es un problema que no se tiene con el atacado químico, ya que la placa es sumergida completamente en acido que corroe todo el cobre expuesto y no el sustrato.

Para superar este problema, Kamp (2010) realizó una prueba de concepto de una aplicación informática denominada "height probing", la cual mide la altura de la superficie de cobre del tablero mediante una acción de "probado" (en inglés probing) y compensa los códigos- $\mathrm{G}$ de las coordenadas del eje $\mathrm{Z}$ de acuerdo a ellas. La medición de altura se hace convirtiendo la máquina fresadora en un máquina de medición de coordenadas (en inglés coordinate measuring machine o CMM). Para esto es necesario montar la fresa (herramienta) que se usará para mecanizar el tablero de circuito y conectar un pin del puerto paralelo de la computadora al tablero de cobre, el cual se monta sobre un material no conductor (tal como tela, madera, fibrán, etc.) para aislarlo eléctricamente de la máquina $\mathrm{CNC}$. Cuando la fresa toca el tablero, hace corto con la superficie a tierra 
(en inglés ground) a través de la máquina y el programa registra que se cerró un contacto y registra la altura en $\mathrm{Z}$ alcanzada (ver Figura 3).

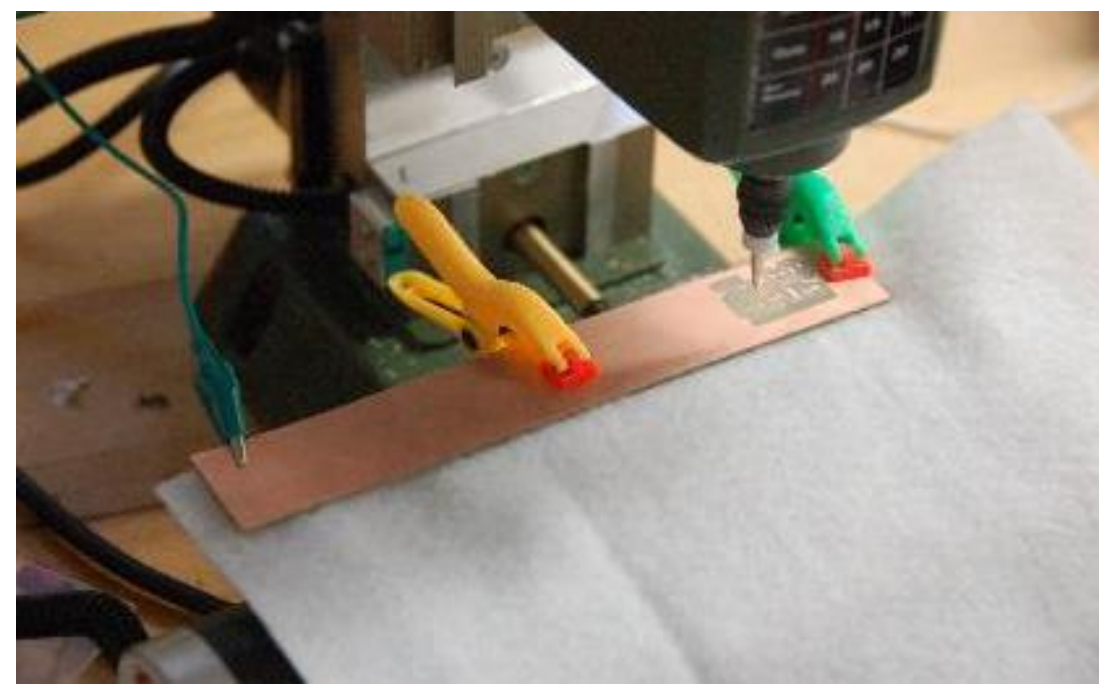

Figura 3. Montaje de un tablero de circuito para la prueba de altura

El programa desarrollado lee el código-G generado por el programa pcb2gcode y separa la trayectoria para poder identificar en qué lugares es necesario identificar las alturas del tablero. Usando una cuadrícula (en inglés grid) se realiza la acción de "probado" en el tablero para medir la altura del área de trabajo y guardar las alturas identificadas en variables de código G. La parte de arriba de la Figura 4 muestra la rutina que se utiliza para realizar el probado. Usando el comando de código-G G38.2, en la posición X y Y especificada por la cuadrícula, se inicia una rutina de descender la herramienta desde una altura $\mathrm{Z}$ y a una velocidad de avance $\mathrm{F}$ hasta tocar la superficie de la placa. Estas alturas encontradas van registrándose para poder usarse posteriormente en un algoritmo que buscará obtener alturas que han sido interpoladas de acuerdo a las alturas de los puntos cercanos medidos con la herramienta informática. Como se puede ver en la parte de abajo de la Figura 4, el algoritmo utiliza estas variables para buscar optimizar la altura que debe de usarse de acuerdo a la distancia proporcional a la que se encuentra el punto en cuestión de cada altura identificada en la cuadrícula.

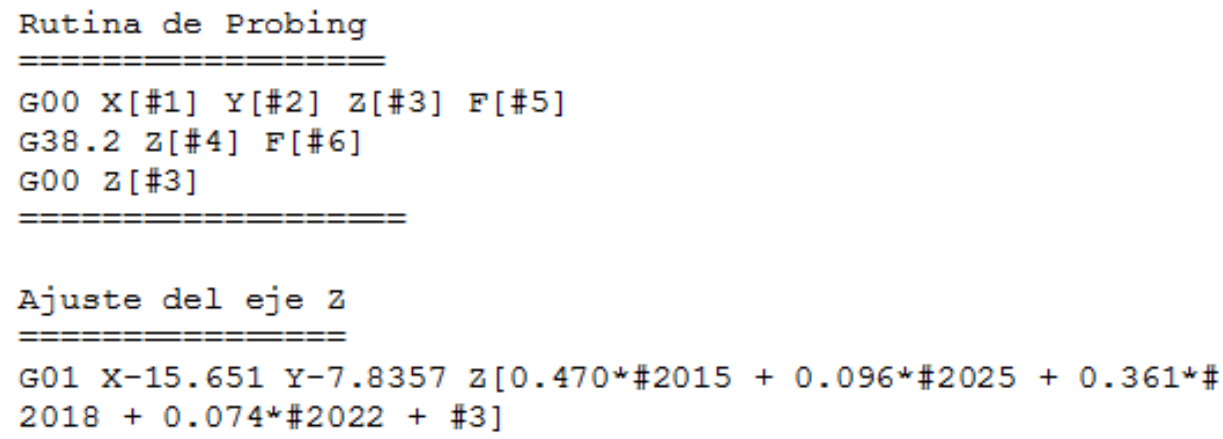

Figura 4. Código conteniendo la rutina de probing y la fórmula de ajuste Z

Este programa fue creado como una prueba de concepto, por lo que no funcionaba completamente y requería de trabajo para volverlo un programa útil y robusto. Sin 
embargo, Kamp dejó disponible su código fuente para que alguien más pudiera seguir desarrollándolo.

\section{Metodología}

Luego de revisar los pasos que son necesarios para manufacturar un tablero de circuitos usando CNC y de ver las áreas en las que esta metodología se podría mejorar, se determinó que era necesario crear una herramienta informática que usara tres elementos de manera integrada:

- Procesado de Gerber a Código-G

- Compensador de alturas del tablero

- Graficador de Código G y cuadrícula

La Figura 5 muestra como estos elementos interactúan entre sí y como lo hacen con el resto de la metodología descrita por Fritz.

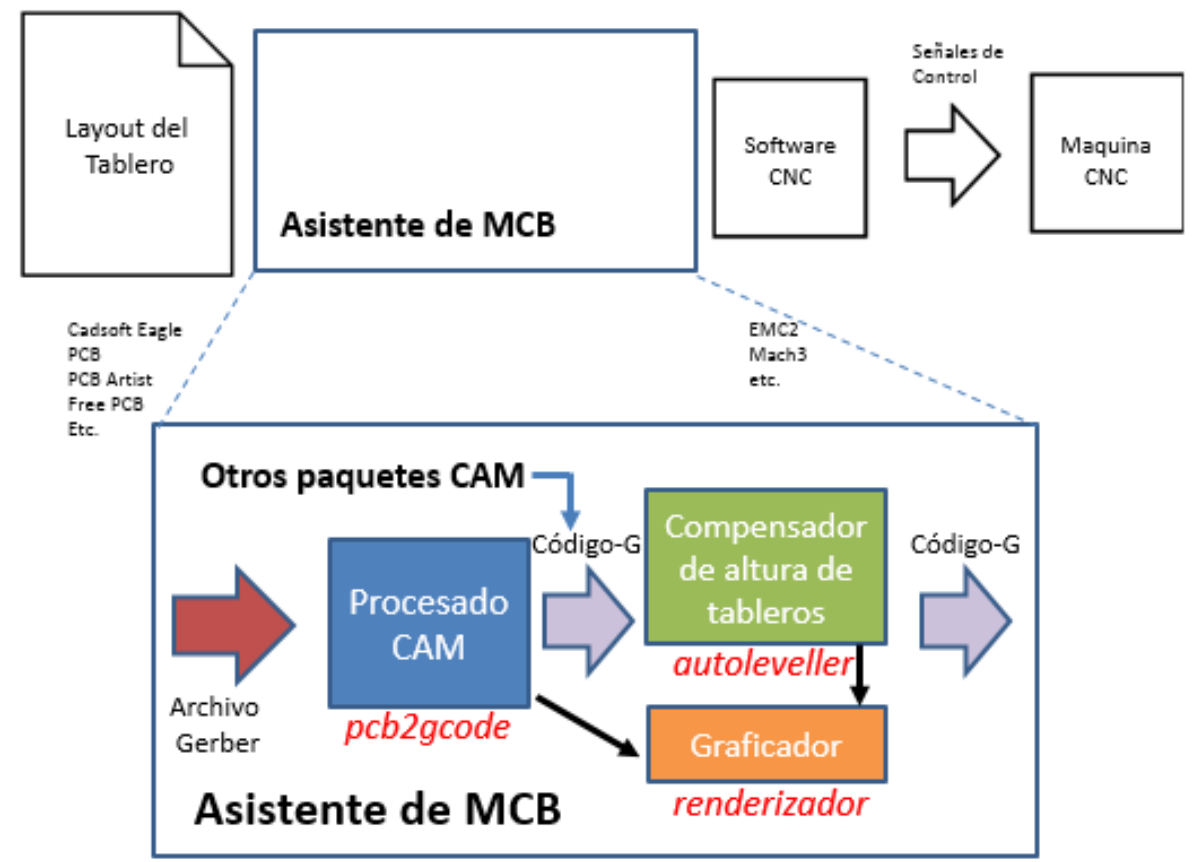

Figura 5. Arquitectura de la herramienta integrada para crear MCBs

En esencia la herramienta integrada desarrollada denominada "Asistente de MCB" permitirá tomar archivos Gerber de circuitos y convertirlos a código-G usando el software "pcb2gcode" para luego, usando el código generado, utilizar el software "autoleveller" para producir un nuevo código-G que tome en consideración y compense por las diferentes alturas del tablero. Ambas herramientas estarán corriendo en el background de un panel de usuario principal que tendría la habilidad de configurar los pasos y rutinas necesarias, y de graficar tanto los toolpaths del código-G como la cuadrícula usada para compensar por las alturas del tablero. Esta herramienta entregará código-G que estará listo para producir MCBs de forma más rápida, exacta y fácil que lo que se puede hacer creando PCBs mediante la técnica tradicional. 


\section{Implementación del Prototipo}

Como se explicó en la metodología, la aplicación integrada requiere de tres aplicaciones que funcionan de manera coordinada, el procesado de archivos Gerber a Código-G, el compensado de las diferentes alturas del tablero y una aplicación que genere un interfaz de usuario y que grafique el Código $\mathrm{G}$ y la cuadrícula.

\subsection{Procesado de Gerber a Código-G}

Es necesario convertir la información del archivo Gerber que describe la forma que deben de tener las pistas e islas del circuito en código-G que pueda ser interpretado y luego mecanizado por la máquina CNC. Para hacer esta conversión se usó el programa pcb2gcode, en su versión escrita en $\mathrm{C}++$. Sin embargo, en vista que este programa fue escrito para ser usado mediante línea de comandos, fue necesario agregarle un interfaz gráfico de usuario (en inglés graphical user interface o GUI) que permitiera interactuar con el de manera más fácil y transparente. Así fue como se creó la aplicación "Gerber to GCode" en la forma de un wizard que es lanzado por la aplicación principal.

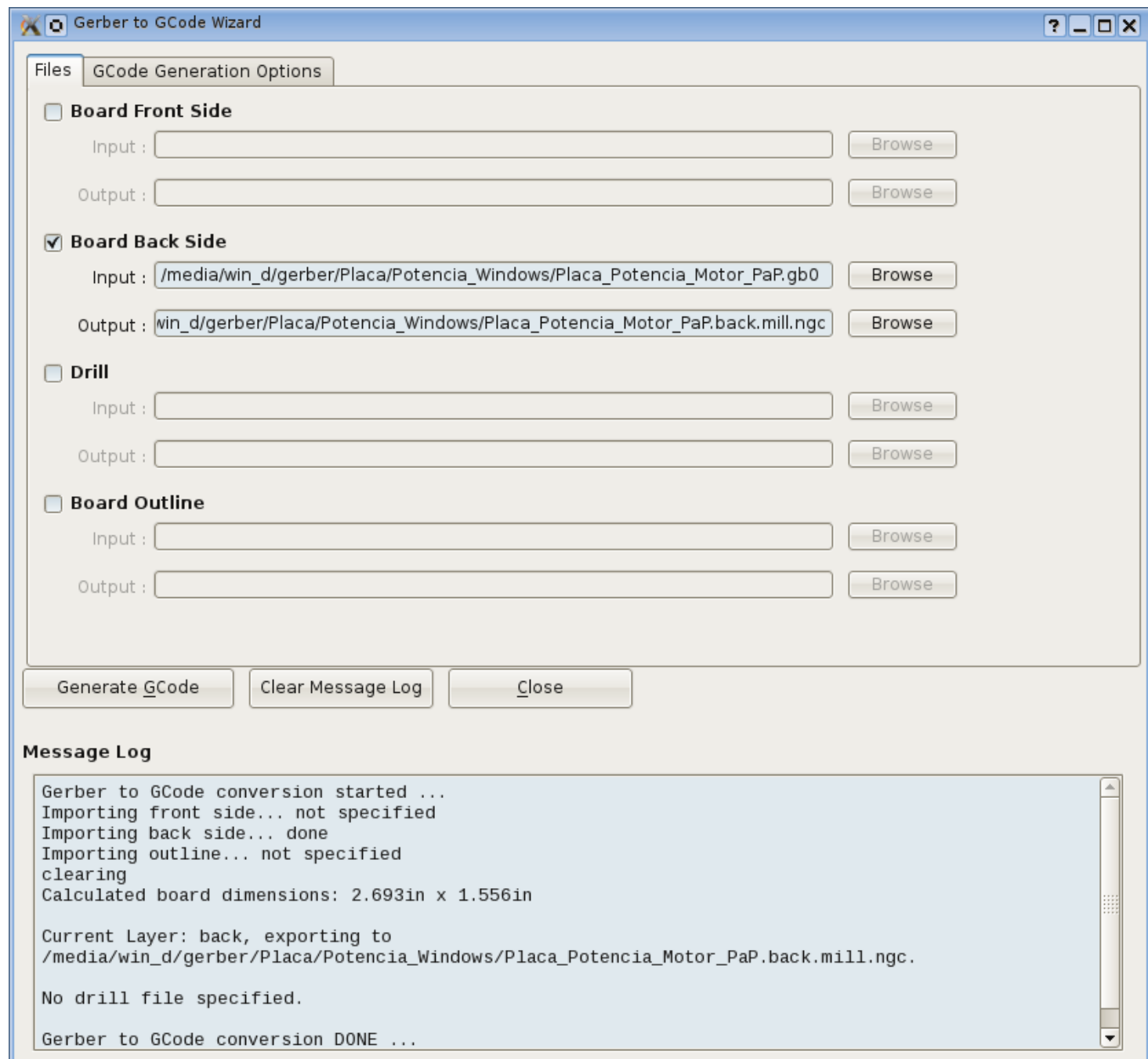

Figura 6. Viñeta "Files" de la pantalla Gerber to GCode 
Como se muestra en la Figura 6, el wizard creado tiene dos viñetas. La primera (llamado "Files) sirve para cargar archivos Gerber que son usados para fabricar tableros de una sola cara (Board Front Side), de dos caras (Board Back Side), con agujeros para colocar componentes (Drill) y para cortar totalmente el tablero (Board Outline). En la parte inferior de la viñeta hay tres botones, "Generate Code" que genera el código-G a partir del archivo Gerber cargado y las variables configuradas. Como se observa, hay en la parte inferior un espacio denominado Message Log en donde se despliega información del estatus de la aplicación. Esta información puede ser borrada usando el botón "Clear Message Log”. El último botón, "Close” cierra el wizard Gerber to GCode. La segunda viñeta (llamada "GCode Generation Options") permite configurar ciertos parámetros necesarios para la generación de código-G tales como las unidades en las que se trabajará (mm o pulgadas), profundidades de corte, velocidad de avance, etc.

La parte gráfica del software fue hecha usando C++ con una librería gráfica llamada QT (Qt Project, 2013). La librería QT facilita la portabilidad de la aplicación desarrollada, ya que puede correr tanto en Windows como en Linux. Para el proceso de conversión de Gerber a GCode la aplicación ejecuta al programa pcb2gcode. Dicha acción implica acumular los argumentos que el usuario configuró en un arreglo llamado "arguments" y después estos son enviados a la aplicación usando el método start de la clase QProcess.

Todo el código usado puede ser visto en www.github.com en ideras/MCB.

\subsection{Compensador de alturas de tableros}

En vista que para realizar MCBs de calidad se necesita compensar en el eje Z por causa de las variaciones en alturas que típicamente tienen los tableros, se decidió tomar como base el programa Height Probing y re-escribirlo pasándolo de $\mathrm{C}$ a $\mathrm{C}++$ para hacerlo más portable y robusto al usar variables dinámicas que permitieran parametrizar el programa. Como se observa en la Figura 7, entre las variables configurables se encuentra el tamaño de la cuadrícula, la profundidad máxima de corte, etc.

Una vez que los parámetros han sido definidos se puede apretar el botón "Autolevel" el cual corre la rutina programada y genera el nuevo código-G modificado en la ruta especificada en el espacio "Output file". La aplicación permite especificar el nombre que recibirá el archivo en código-G que será creado y que contendrá las alturas modificadas o usar un nombre por defecto que está basado en el nombre del archivo de entrada.

La lógica de programación del Autoleveller está dividida en dos secciones, la primera realiza un análisis de todo el archivo para lograr dividir todos los segmentos presentes en partes más pequeñas que no pueden ser más grandes que el tamaño de la cuadrícula. Como se observa en la Figura 8, la segunda parte consiste en crear los puntos de la cuadrícula en función de las posiciones en donde están ubicados los segmentos, de forma que no sea necesario llenar todo el tablero de puntos, sino solo aquellos lugares en donde hay segmentos que requieren ajuste de altura. 


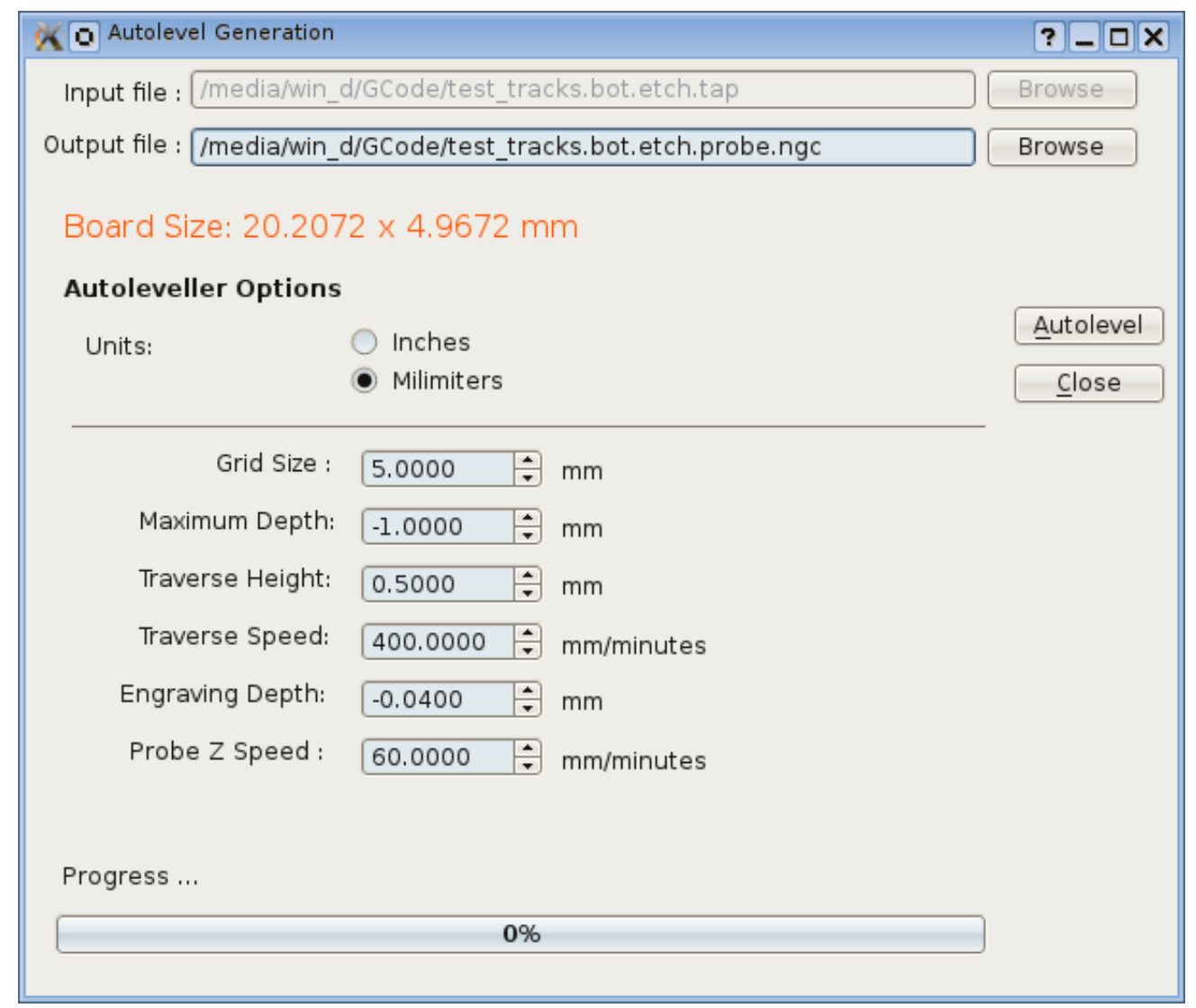

Figura 7. Pantalla del programa Autoleveller para compensar altura en $\mathrm{Z}$

La tercera parte es ejecutar la rutina de medir las alturas usando como referencias los puntos calculados. Para esto es necesario generar código-G que mueve la herramienta a los puntos generados y mide la altura de la placa usando el comando G38.2. La lectura da una señal TTL por parte del puerto paralelo de la computadora, la cual se activa cada vez que la herramienta (conectado a tierra) toca el tablero (pin de entrada), envía la señal y registra la altura obtenida. El último paso consiste en interpolar para cada segmento el valor de altura $\mathrm{Z}$ que se usará en el ajuste de la profundidad de corte a usar en función de las alturas obtenidas en los puntos de la cuadrícula que fueron creados. Estas alturas, que fueron guardadas en un set de variables, son en este momento actualizadas y colocadas en el nuevo código-G generado.

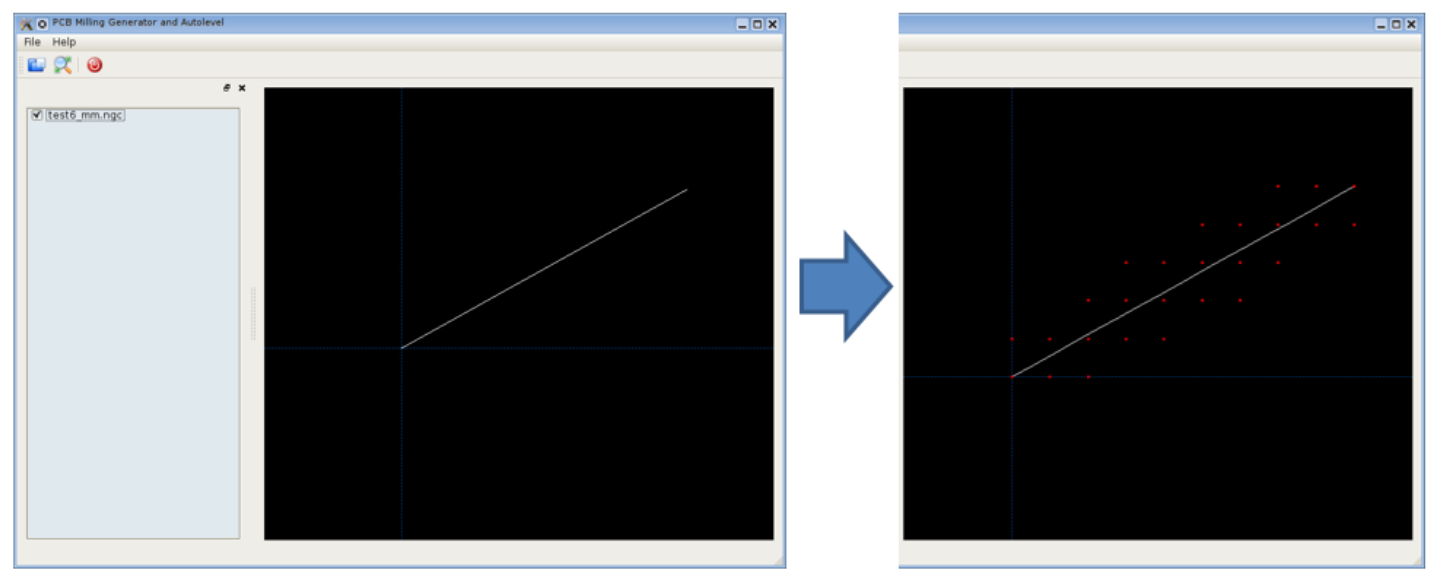

Figura 8. Creación de puntos de cuadricula para medir alturas 


\subsection{Graficador de Código-G y cuadricula}

En vista que era necesario tener una pantalla principal de la aplicación desarrollada que integrará las dos herramientas antes mencionadas, se decidió crear un interfaz que permitiera el acceso a configurar parámetros, tuviera la habilidad de leer el código $\mathrm{G}$ generado ya sea por pcb2gcode o autoleveller, y permitiera graficar las rutas producidas de manera que el usuario pudiera visualizarlas fácilmente. Al igual que con el resto de aplicaciones, esta se hizo usando la librería QT (ver Figura 9).

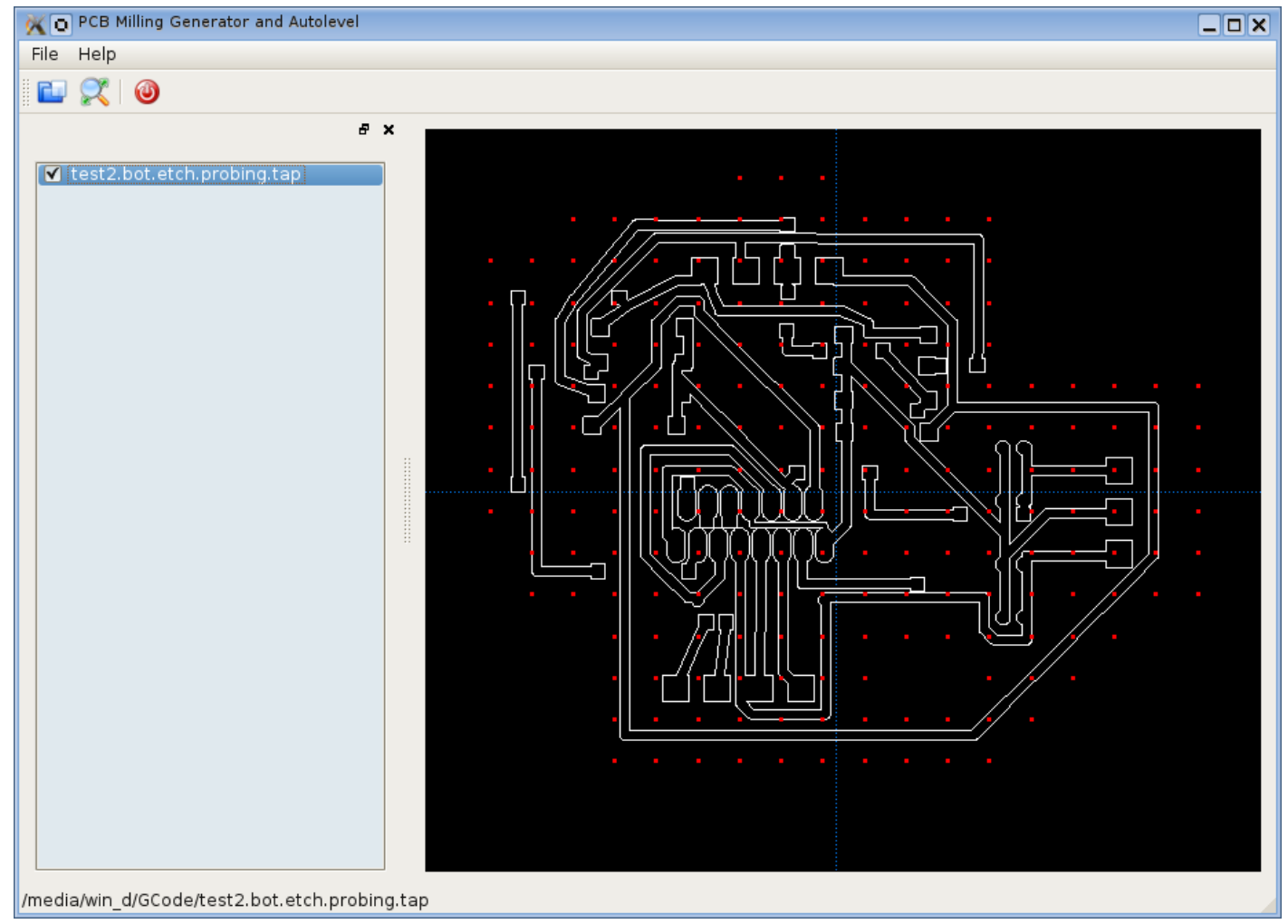

Figura 9. Ejemplo de código-G y cuadricula renderizada en el Asistente de MCB

\subsection{Prototipo de tablero de circuito MCB}

Utilizando el Asistente de MCB desarrollado, se procedió a seguir los pasos antes descritos para crear un tablero de circuito usando una máquina $\mathrm{CNC}$. Se diseñó el circuito a fabricar y se creó su correspondiente archivo Gerber usando el software para creación de PCBs CadSoft Eagle. Este archivo fue ingresado en el Asistente de MCB y se procedió a mecanizar el tablero de circuito. Para esto se usó la máquina fresadora CNC Sherline que se encuentra en el laboratorio de máquinas y herramientas de Unitec campus San Pedro Sula. Como se puede ver en la Figura 10, se logró manufacturar un tablero de alta calidad, en un tiempo de 28 minutos de manera relativamente sencilla. La velocidad de maquinado utilizada fue de 3 pulgadas por minuto (IPM), esto debido a que a que la velocidad de rotación del husillo (spindle) alcanza un máximo de 3000 rotaciones por minuto (RPM). 


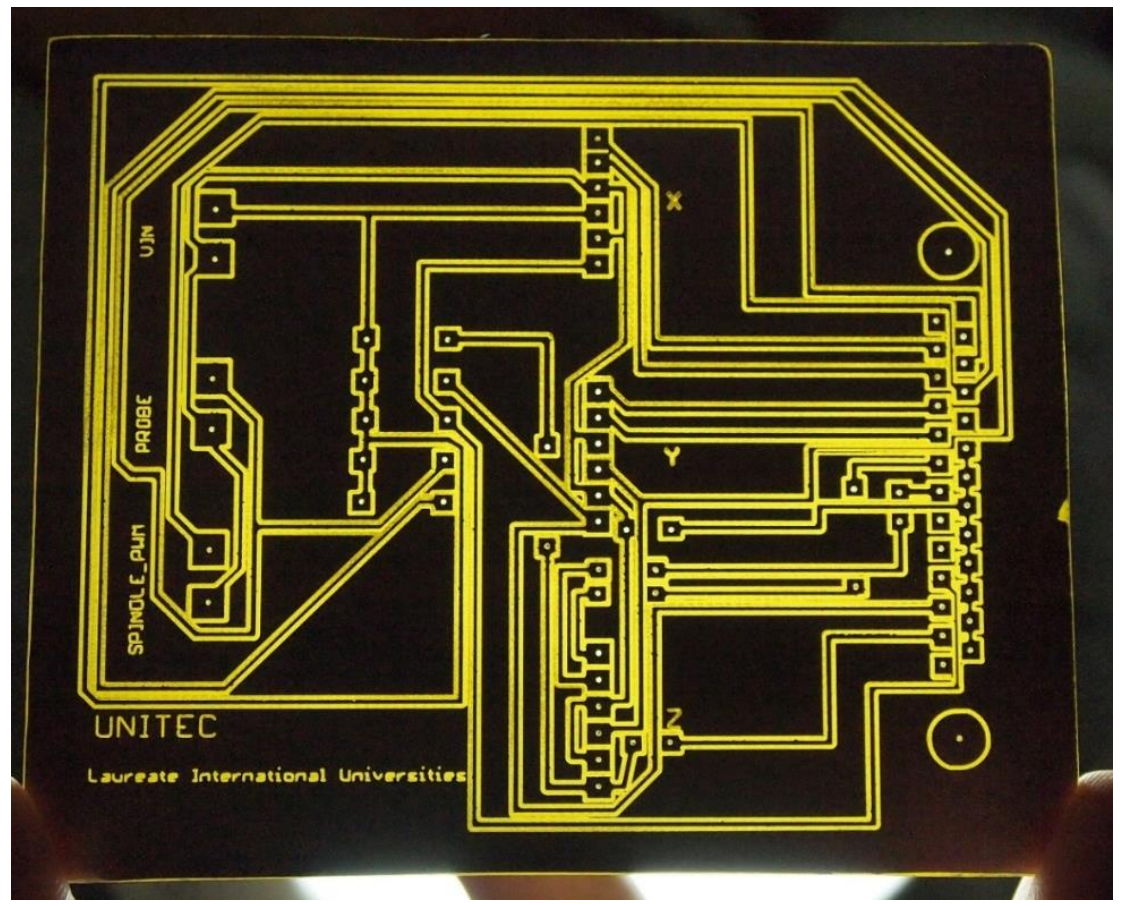

Figura 10. Tablero de circuito producido usando el Asistente de MCB

Adicionalmente se realizó una prueba para revisar la calidad de los tableros obtenidos usando la técnica MCB y PCB y el tiempo de fabricación que tomó realizar con cada una de ellas. Como se puede observar en la Figura 11, la calidad del acabado de la MCB (parte superior de la imagen) es bastante superior a la de la PCB (parte inferior). Igualmente el tiempo de fabricación de la MCB fue de solo 5 minutos, mientras el proceso de la PCB fue de 35 minutos. De acuerdo a este único ejemplo pareciera que las hipótesis planteadas son correctas, aunque se requiere de más experimentación para validarlas estadísticamente.

\section{MCB}
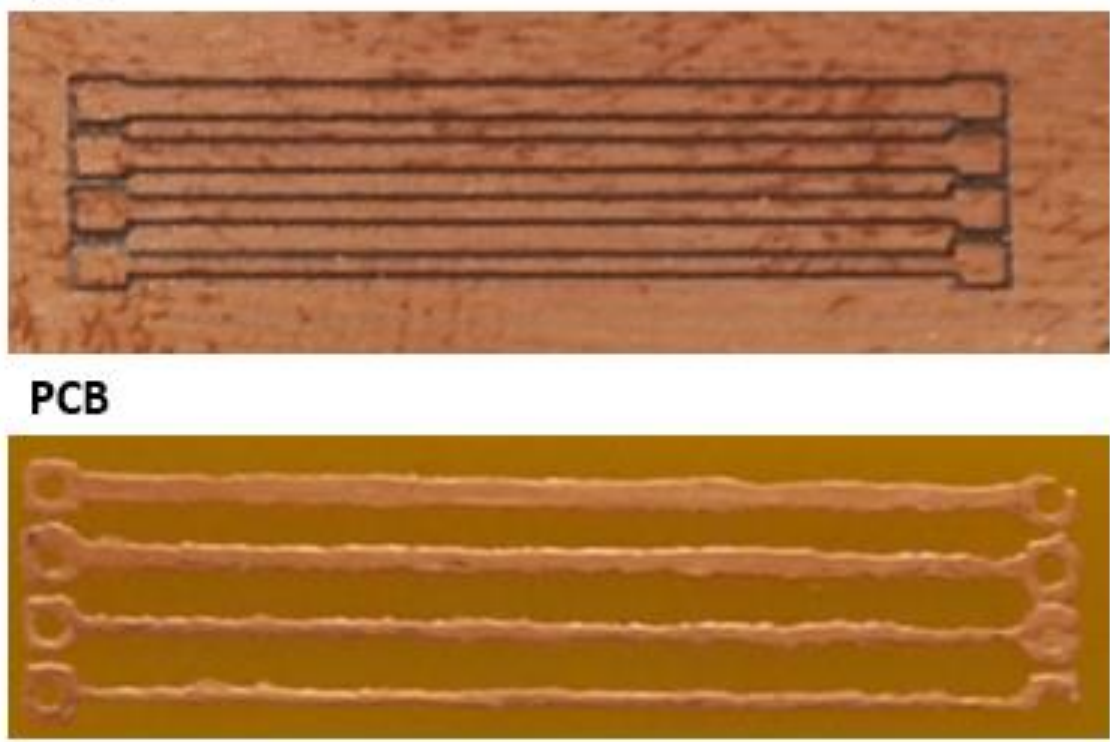

Figura 11. Pruebas de calidad y tiempo de MCB y PCB 


\section{Conclusiones}

En este documento se presentó el desarrollo e implementación de un sistema integrado para la creación de tableros de circuitos de alta calidad y al mismo tiempo fácil de implementar, que no utiliza ácidos nocivos para la salud y el medioambiente. La aplicación denominada Asistente de MCB permite tomar archivos Gerber con el diseño de un circuito y crear tableros de circuito que toman en consideración las diferentes alturas, que por problemas de alineación tiene el tablero. El proyecto se realizó combinando la herramienta existente pcb2gcode, modificando la aplicación Height Probing y convirtiéndola en el programa Autolevelling y creando una nueva aplicación que integraba estas dos herramienta y agregaba la habilidad de graficar toolpaths y la cuadrícula. Se hicieron pruebas de fabricación que mostraron que el asistente desarrollado era capaz de crear tableros de circuitos de alta calidad.

El sistema puede mejorarse mucho con algunas recomendaciones:

1. La aplicación pcb2gcode genera código $G$ no optimizado, es decir genera toolpaths que saltan de un extremo a otro de la placa. Se sugiere optimizar el código $\mathrm{G}$ generado de forma que se pueda minimizar la distancia de salto entre toolpaths.

2. La máquina $\mathrm{CNC}$ utilizada en las pruebas no contaba con un spindle rápido (que rote a más de 10K RPM). Se sugiere hacer más pruebas utilizando un spindle más rápido. Esto, junto con la optimización de los toolpaths, podría reducir aún más el tiempo de maquinado de un tablero de circuito maquinado en la CNC.

3. Se sugiere validar las hipótesis de que MCB genera tableros de mejor calidad y en un tiempo menor que PCB realizando experimentos con más repeticiones de forma que sean estadísticamente correctos. Esto permitirá saber si en efecto el Asistente de MCB es una mejor alternativa al método tradicional de fabricación de los tableros de circuitos que deben preparar los estudiantes de Unitec.

\section{Bibliografía}

Bonwell, C. C., \& James, E. J. (1991). Active Learning: Creating Excitment in the Clasroom. ASHE-ERIC Higher Education Report No. 1.

Devadoss, S., \& Foltz, J. (1996). Evaluation of Factors Influencing Student Class Attendance and Performance. American Journal of Agriculture Economics, Vol. 78, No. 3, 499 - 507.

Fritz, T. (2010, Marzo 17). coldelectrons.com. From Bots-N-Copper: Milling Circuit Boards: http://www.coldelectrons.com/talks/Notacon/2010/botsncopper.html

Groover, M. P. (1997). Fundamentos de Manufactura Moderna. Ciudad de Mexico: Prentice-Hall Hispanoamerica, S.A.

Kamp, P.-H. (2010, Abril 4). Height probing for PCB isolation routing with Eagle/pcbgcode. From http://phk.freebsd.dk/CncPcb/ 
Lynch, M. (2013, Septiembre 18). CNC Concepts Inc. From Training Materiales for CNC: http://www.cncci.com/resources/articles/what\%20is\%20cnc.htm

Major, C. H., \& Palmer, B. (2001). Assesing the Effectiveness of Problem-Based Learning in Higher Education: Lessons from the Literature. Academic Exchange Quarterly, Vol 5, No 1.

Qt Project. (2013, Septiembre 28). From http://qt-project.org/

Rey, F. (2013, Septiembre 18). Como hacer una placa de circuito impreso. From http://usuaris.tinet.cat/fmco/download/Tutorial_placas.pdf

SourceForge. (2011, Diciembre 27). From pcbtogcode: http://sourceforge.net/apps/mediawiki/pcb2gcode/ index.php?title=Main_Page

UNESCO. (2010). UNESCO Science Report 2010: The Current Status of Science around the World. Paris: UNESCO Publishing.

Wee, C. (2013, Febrero 4). What is CDIO? From Singapure Polytechnic: http://educator.sp.edu/cdio/what-is-cdio/

Williams, A. (2004). Build your own printed circuit board. McGraw-Hill Professional.

"LA REVISTA INNOVARE NO SE HACE RESPONSABLE EN NINGÚN CASO DE LOS CONTENIDOS, DATOS, CONCLUSIONES U OPINIONES VERTIDAS EN LOS ARTÍCULOS PUBLICADOS, SIENDO ESTA RESPOSABILIDAD EXCLUSIVA DEL (DE LOS) AUTOR (AUTORES)" 